\title{
Overdose Deaths Involving Opioids, Cocaine, and Psychostimulants - United States, 2015-2016
}

\author{
Puja Seth, $\mathrm{PhD}^{1}$; Lawrence Scholl, $\mathrm{PhD}^{1,2}$; Rose A. Rudd, MSPH ${ }^{1}$; Sarah Bacon, $\mathrm{PhD}^{1}$
}

During 1999-2015, 568,699 persons died from drug overdoses in the United States.* Drug overdose deaths in the United States increased 11.4\% from 2014 to 2015 resulting in 52,404 deaths in 2015, including 33,091 (63.1\%) that involved an opioid. The largest rate increases from 2014 to 2015 occurred among deaths involving synthetic opioids other than methadone (synthetic opioids) (72.2\%) (1). Because of demographic and geographic variations in overdose deaths involving different drugs $(2,3),{ }^{\dagger} \mathrm{CDC}$ examined age-adjusted death rates for overdoses involving all opioids, opioid subcategories (i.e., prescription opioids, heroin, and synthetic opioids), ${ }^{\S}$ cocaine, and psychostimulants with abuse potential (psychostimulants) by demographics, urbanization levels, and in 31 states and the District of Columbia (DC). There were 63,632 drug overdose deaths in 2016; 42,249 (66.4\%) involved an opioid. ' From 2015 to 2016, deaths increased across all drug categories examined. The largest overall rate increases occurred among deaths involving cocaine (52.4\%) and synthetic opioids (100\%), likely driven by illicitly manufactured fentanyl (IMF) $(2,3)$. Increases were observed across demographics, urbanization levels, and states and DC. The opioid overdose epidemic in the United States continues to worsen. A multifaceted approach, with faster and more comprehensive surveillance, is needed to track emerging threats to prevent and respond to the overdose epidemic through naloxone availability, safe prescribing practices, harm-reduction services, linkage into

\footnotetext{
*https://wonder.cdc.gov.

$\dagger$ https://www.cdc.gov/drugoverdose/pdf/pubs/2017-cdc-drug-surveillancereport.pdf.

${ }^{\S}$ Natural opioids include morphine and codeine, and semisynthetic opioids include drugs such as oxycodone, hydrocodone, hydromorphone, and oxymorphone. Methadone is a synthetic opioid. Synthetic opioids, other than methadone, include drugs such as tramadol and fentanyl. Heroin is an illicit opioid synthesized from morphine that can be a white or brown powder, or a black sticky substance.

I https://www.cdc.gov/nchs/products/databriefs/db294.htm.
}

treatment, and more collaboration between public health and public safety agencies.

Drug overdose deaths were identified in the National Vital Statistics System multiple cause-of-death mortality files, ${ }^{* *}$ using the International Classification of Diseases, Tenth Revision (ICD-10), based on ICD-10 underlying cause-of-death codes X40-44 (unintentional), X60-64 (suicide), X85 (homicide), or Y10-Y14 (undetermined intent). Among deaths with drug overdose as the underlying cause, the type of drug or drug category is indicated by the following ICD-10 multiple cause-of-death codes: opioids (T40.0, T40.1, T40.2, T40.3,

** https://www.cdc.gov/nchs/nvss/mortality_public_use_data.htm.

\section{INSIDE}

359 Prevalence of Diagnosed Diabetes in Adults by Diabetes Type — United States, 2016

362 Trends in Diabetic Ketoacidosis Hospitalizations and In-Hospital Mortality — United States, 2000-2014

366 Two Cases of Meningococcal Disease in One Family Separated by an Extended Period - Colorado, 2015-2016

369 Notes from the Field: Nontuberculous Mycobacteria Infections in U.S. Medical Tourists Associated with Plastic Surgery — Dominican Republic, 2017

371 Notes from the Field: Fatalities Associated with Human Adenovirus Type 7 at a Substance Abuse Rehabilitation Facility - New Jersey, 2017

374 QuickStats

Continuing Education examination available at https://www.cdc.gov/mmwr/cme/conted_info.html\#weekly.

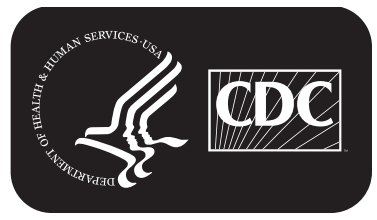

U.S. Department of Health and Human Services Centers for Disease Control and Prevention 
T40.4, or T40.6) ${ }^{\dagger \dagger}$; natural/semisynthetic opioids (T40.2); methadone (T40.3); heroin (T40.1); synthetic opioids other than methadone (T40.4); cocaine (T40.5); and psychostimulants with abuse potential (T43.6). Some deaths involved more than one type of drug; these deaths were included in the rates for each drug category. Therefore, categories are not mutually exclusive. $\$ \mathbb{\$}$

Age-adjusted overdose death rates $\$$ were examined for 2015 and 2016 for all opioids, opioid subcategories (prescription opioids [i.e., natural/semisynthetic opioids and methadone] (4), heroin, and synthetic opioids), cocaine, and psychostimulants in the United States and by age, sex, racial/ethnic group, urbanization level, ${ }^{* * *}$ and state. State-level analyses included

i† T40.0 (opium) and T40.6 (other and unspecified narcotics).

$\$ \$$ For example, a death involving both a synthetic opioid other than methadone and heroin would be included in both the synthetic other than methadone and heroin death rates.

99 Age-adjusted death rates were calculated by applying age-specific death rates to the 2000 U.S. Census standard population age distribution. https://www. cdc.gov/nchs/data/nvsr/nvsr61/nvsr61_04.pdf.

*** Categories of 2013 NCHS Urban-Rural Classification Scheme for Counties (https://www.cdc.gov/nchs/data_access/urban_rural.htm): Large central metro: Counties in metropolitan statistical areas (MSAs) of $\geq 1$ million population that 1) contain the entire population of largest principal city of the MSA, or 2) have their entire population contained in the largest principal city of the MSA, or 3) contain at least 250,000 inhabitants of any principal city of the MSA; Large fringe metro: Counties in MSAs of $\geq 1$ million population that did not qualify as large central metro counties; Medium metro: Counties in MSAs of populations of 250,000-999,999; Small metro: Counties in MSAs of populations <250,000; Micropolitan (nonmetropolitan counties): counties in micropolitan statistical areas; Noncore (nonmetropolitan counties): nonmetropolitan counties that did not qualify as micropolitan.
31 states and DC that met the following criteria: 1$) \geq 80 \%$ of drug overdose death certificates named at least one specific drug in 2015 and 2016; 2) change from 2015 to 2016 in the percentage of death certificates reporting at least one specific drug was $<10$ percentage points ${ }^{\dagger \dagger}$; and 3) $\geq 20$ deaths occurred during 2015 and 2016 in at least two drug categories examined. These inclusion criteria were selected to ensure accurate examination of death rates and increases. Relative change in age-adjusted rates and absolute change were calculated. Significance was assessed using z-tests when the number of deaths was $\geq 100$ $(\mathrm{p}<0.05)$ and nonoverlapping confidence intervals based on a gamma distribution when the number of deaths was $<100$. $\$ \$ \$ \$ S$

In the United States, 63,632 drug overdose deaths occurred in 2016; the age-adjusted rate of overdose deaths increased significantly (21.5\%) from 16.3 in 2015 to 19.8 in 2016. Opioids were involved in 42,249 (66.4\%) drug overdose deaths (13.3 per 100,000 population) in 2016, representing a $27.9 \%$ rate increase from 2015 (Table 1). These increases primarily

\footnotetext{
I†† States whose reporting of any specific drug or drugs involved in an overdose changed by $\geq 10$ percentage points from 2015 to 2016 were excluded because drug-specific overdose numbers and rates might have changed substantially from 2015 to 2016 as a result of changes in reporting.

$\$ \$ \$$ Z-tests were used if the number of deaths was $\geq 100$, and a p-value of $<0.05$ was considered to be statistically significant. Nonoverlapping confidence intervals based on the gamma method were used if the number of deaths was $<100$ in 2015 or 2016 . Note that the method of comparing confidence intervals is a conservative method for statistical significance; caution should be observed when interpreting a nonsignificant difference when the lower and upper limits being compared overlap only slightly.
}

The MMWR series of publications is published by the Center for Surveillance, Epidemiology, and Laboratory Services, Centers for Disease Control and Prevention (CDC), U.S. Department of Health and Human Services, Atlanta, GA 30329-4027.

Suggested citation: [Author names; first three, then et al., if more than six.] [Report title]. MMWR Morb Mortal Wkly Rep 2018;67:[inclusive page numbers].

\section{Centers for Disease Control and Prevention}

Robert R. Redfield, MD, Director

Anne Schuchat, MD, Principal Deputy Director

Leslie Dauphin, PhD, Acting Associate Director for Science

Joanne Cono, MD, ScM, Director, Office of Science Quality

Chesley L. Richards, MD, MPH, Deputy Director for Public Health Scientific Services

Michael F. Iademarco, MD, MPH, Director, Center for Surveillance, Epidemiology, and Laboratory Services

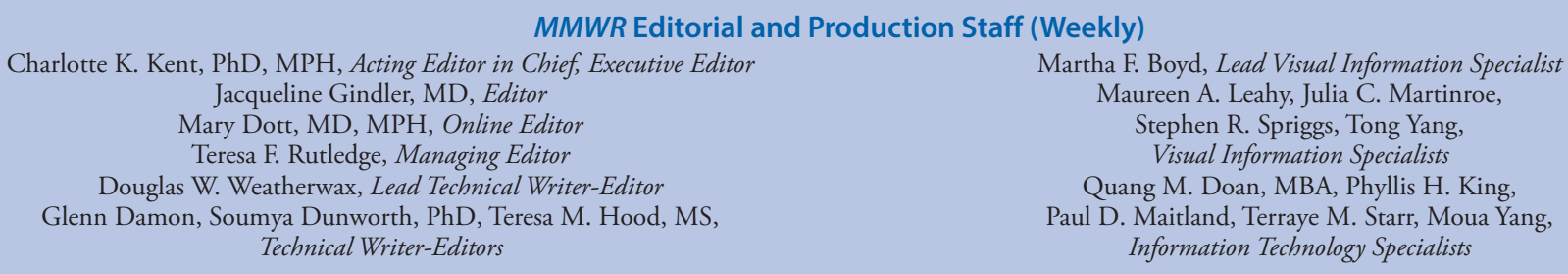

MMWR Editorial Board

Timothy F. Jones, MD, Chairman

Matthew L. Boulton, MD, MPH

Virginia A. Caine, MD

Katherine Lyon Daniel, PhD

Jonathan E. Fielding, MD, MPH, MBA

David W. Fleming, MD
William E. Halperin, MD, DrPH, MPH

King K. Holmes, MD, PhD

Robin Ikeda, MD, MPH

Rima F. Khabbaz, MD

Phyllis Meadows, PhD, MSN, RN

Jewel Mullen, MD, MPH, MPA
Jeff Niederdeppe, $\mathrm{PhD}$

Patricia Quinlisk, MD, MPH

Patrick L. Remington, MD, MPH Carlos Roig, MS, MA

William L. Roper, MD, MPH

William Schaffner, MD 
were driven by deaths involving synthetic opioids, for which the rate doubled from 2015 to 2016 (Table 2). Rates of overdose deaths involving prescription opioids and heroin increased by $10.6 \%$ and $19.5 \%$, respectively (Table 1) (Table 2), and rates of overdose deaths involving cocaine and psychostimulants increased by $52.4 \%$ and $33.3 \%$, respectively (Table 3 ).

From 2015 to 2016, opioid-involved deaths increased in males and females and among persons aged $\geq 15$ years, whites, blacks, Hispanics, and Asian/Pacific Islanders. The largest relative rate change occurred among blacks (56.1\%) (Table 1). The largest absolute rate increases of opioid-involved deaths and deaths involving synthetic opioids occurred among males aged 25-44 years and persons aged 25-34 years. However, deaths involving synthetic opioids increased in every subgroup examined (Table 2). Rates involving prescription opioids, heroin, cocaine, and psychostimulants increased for both sexes, whites, blacks, and most age groups (Table 1) (Table 2) (Table 3). Counties in large central and fringe metro areas experienced the largest absolute increases in deaths involving prescription and synthetic opioids, heroin, and cocaine; micropolitan areas experienced the largest increase in rates involving psychostimulants (Table 1) (Table 2) (Table 3).

Opioid death rates differed across the 31 states and DC, with synthetic opioids driving increases in many states. 999 Although several states experienced increases across drug categories, in many, the changes from 2015 to 2016 were not significant. Rates of deaths involving synthetic opioids ranged from 0.9 to 30.3 per 100,000, with the largest rates and increases concentrated in eastern states. New Hampshire (30.3 per 100,000), West Virginia (26.3), and Massachusetts (23.5) had the highest synthetic opioid death rates. Twenty states and DC experienced increases in overdose death rates involving synthetic opioids, with 10 experiencing increases by $\geq 100 \%$; the largest such increase $(392.3 \%)$ occurred in DC, followed by Illinois (227.3\%) and Maryland (206.9\%) (Table 2). Many states with large increases in synthetic opioid death rates also had large increases in rates involving other drug categories (e.g., Maryland, Virginia, and DC), including any opioid, prescription opioids (Table 1), heroin (Table 2), and cocaine (Table 3).

Thirteen states and DC experienced significant increases in heroin-involved death rates, whereas a significant decrease (56.9\%) occurred in New Hampshire (Table 2). In 2016, the highest rates were in DC (17.3 per 100,000), West Virginia (14.9), and Ohio (13.5). The rates of prescription opioidinvolved overdose deaths significantly increased in seven

\footnotetext{
999 Maps and figures providing significant changes in drug overdose and opioidinvolved overdose death rates by state are available on CDC's Drug Overdose website: https://www.cdc.gov/drugoverdose/data.
}

states and DC, with the highest rates in West Virginia (19.7), Maryland (13.1), Maine (12.5), and Utah (12.5) (Table 1). The highest cocaine-involved overdose death rates occurred in DC (13.5), Rhode Island (10.7), and Ohio (10.1), with 15 states and DC experiencing a significant increase from 2015 (Table 3). Significant increases in overdose death rates from heroin, prescription opioids, and cocaine occurred primarily in states in the eastern part of the country. Fourteen states experienced significant increases in psychostimulant-involved overdose death rates. The highest rates were in midwestern and western states: Nevada (7.5), New Mexico (7.1), and Oklahoma (7.1) (Table 3).

\section{Discussion}

Drug overdoses resulted in 632,331 deaths from 1999 to 2016 in the United States, with 351,630 being opioid overdose deaths. ${ }^{* * * *}$ The epidemic has continued to worsen, with deaths increasing from 2015 to 2016 across all drug categories examined. Opioid-involved overdoses accounted for two thirds of drug overdose deaths, with increases across age and racial/ ethnic groups, urbanization levels, and in numerous states. The findings highlight wide state and regional variations. Some states (e.g., New Hampshire, Ohio, and West Virginia,) experienced the highest overdose death rates across multiple drug categories, and others (primarily in the Midwest and West) recorded the highest rates of psychostimulant-involved overdose deaths. In New Hampshire, although heroin-involved death rates declined from 2015 to 2016, deaths involving synthetic opioids increased, as they did in most states. In addition, in some states (e.g., Maryland, Rhode Island, and West Virginia), 2016 rates of prescription opioid-involved deaths were higher than were those involving heroin. These data highlight the persistent and multifaceted nature of overdoses.

The first wave of opioid overdose deaths began in the 1990s and included prescription opioid deaths. ${ }^{\dagger \dagger \dagger}$ A second wave, which began in 2010, was characterized by heroin deaths (5). A third wave started in 2013, with deaths involving highly potent synthetic opioids, particularly IMF and fentanyl analogs $(2,3,6) . \$ \$ \$ \$$ Synthetic opioid-involved deaths in 2016 accounted for $30.5 \%$ of all drug overdose deaths and $45.9 \%$ of all opioid-involved deaths, with a $100 \%$ increase in the rate of these deaths compared with 2015. Synthetic opioids propelled increases with 19,413 deaths (more than any drug examined), and previous findings underscore the contribution of IMF. In addition, IMF is now being mixed into counterfeit

\footnotetext{
**** https://www.cdc.gov/nchs/data/databriefs/db294_table.pdf.

$\dagger_{\dagger \dagger \dagger}$ https://www.cdc.gov/nchs/products/databriefs/db81.htm.

$\$ \$ \$ \$ \$ 2017$ National Drug Threat Assessment: https://www.dea.gov/docs/DIR040-17_2017-NDTA.pdf; https://emergency.cdc.gov/han/han00384.asp; https://emergency.cdc.gov/han/han00395.asp.
} 
TABLE 1. Annual number and age-adjusted rate of drug overdose deaths* involving any opioid ${ }^{\dagger}$ and prescription opioids,, , ${ }^{\circ}$ by sex, age, race and Hispanic origin, ${ }^{* *}$ urbanization level, ${ }^{\dagger \dagger}$ and selected states ${ }^{\S \S}$ — United States, 2015 and 2016

\begin{tabular}{|c|c|c|c|c|c|c|c|c|c|c|c|c|}
\hline \multirow[b]{3}{*}{ Decedent characteristic } & \multicolumn{6}{|c|}{ Opioids } & \multicolumn{6}{|c|}{ Prescription opioids } \\
\hline & \multicolumn{2}{|c|}{2015} & \multicolumn{2}{|c|}{2016} & \multicolumn{2}{|c|}{$\begin{array}{l}\text { Change from } \\
2015 \text { to } 2016 \text { ๆी }\end{array}$} & \multicolumn{2}{|c|}{2015} & \multicolumn{2}{|c|}{2016} & \multicolumn{2}{|c|}{$\begin{array}{l}\text { Change from } \\
2015 \text { to } 2016^{\text {ๆी }}\end{array}$} \\
\hline & No. & Rate & No. & Rate & $\begin{array}{c}\text { Absolute } \\
\text { rate change }\end{array}$ & $\begin{array}{l}\% \text { Change } \\
\text { in rate }\end{array}$ & No. & Rate & No. & Rate & $\begin{array}{c}\text { Absolute } \\
\text { rate change }\end{array}$ & $\begin{array}{c}\% \text { Change } \\
\text { in rate }\end{array}$ \\
\hline All & 33,091 & 10.4 & 42,249 & 13.3 & $2.9 * * *$ & $27.9 * * *$ & 15,281 & 4.7 & 17,087 & 5.2 & $0.5^{* * * *}$ & $10.6^{* * *}$ \\
\hline \multicolumn{13}{|l|}{ Sex } \\
\hline Male & 21,671 & 13.7 & 28,498 & 18.1 & $4.4^{* * *}$ & $32.1^{* * *}$ & 8,617 & 5.4 & 9,978 & 6.2 & $0.8^{* * *}$ & $14.8^{* * *}$ \\
\hline Female & 11,420 & 7.1 & 13,751 & 8.5 & $1.4^{* * *}$ & $19.7^{* * *}$ & 6,664 & 4.0 & 7,109 & 4.3 & $0.3^{* * *}$ & $7.5^{* * *}$ \\
\hline \multicolumn{13}{|l|}{ Age group (yrs) } \\
\hline $0-14$ & 83 & 0.1 & 83 & 0.1 & 0.0 & 0.0 & 61 & 0.1 & 60 & 0.1 & 0.0 & 0.0 \\
\hline $15-24$ & 3,082 & 7.0 & 4,027 & 9.3 & $2.3^{* * *}$ & $32.9 * * *$ & 886 & 2.0 & 1,146 & 2.6 & $0.6^{* * *}$ & $30.0^{* * *}$ \\
\hline $25-34$ & 8,568 & 19.4 & 11,552 & 25.9 & $6.5^{* * *}$ & $33.5^{* * *}$ & 2,906 & 6.6 & 3,442 & 7.7 & $1.1^{* * *}$ & $16.7^{* * *}$ \\
\hline $35-44$ & 7,484 & 18.4 & 9,747 & 24.1 & $5.7^{* * *}$ & $31.0^{* * *}$ & 3,390 & 8.4 & 3,727 & 9.2 & $0.8^{* * * *}$ & $9.5^{* * *}$ \\
\hline $45-54$ & 7,595 & 17.6 & 9,074 & 21.2 & $3.6^{* * *}$ & $20.5^{* * *}$ & 4,100 & 9.5 & 4,307 & 10.1 & $0.6^{* * *}$ & $6.3^{* * *}$ \\
\hline $55-64$ & 5,089 & 12.4 & 6,321 & 15.2 & $2.8^{* * *}$ & $22.6^{* * *}$ & 3,101 & 7.6 & 3,489 & 8.4 & $0.8^{* * *}$ & $10.5^{* * *}$ \\
\hline$\geq 65$ & 1,188 & 2.5 & 1,441 & 2.9 & $0.4^{* * *}$ & $16.0^{* * *}$ & 835 & 1.7 & 915 & 1.9 & $0.2^{* * *}$ & $11.8^{* * *}$ \\
\hline \multicolumn{13}{|l|}{ Sex and age group (yrs) } \\
\hline \multicolumn{13}{|l|}{ Male } \\
\hline $15-24$ & 2,211 & 9.8 & 2,986 & 13.4 & $3.6^{* * *}$ & $36.7^{* * *}$ & 619 & 2.8 & 852 & 3.8 & $1.0^{* * *}$ & $35.7^{* * *}$ \\
\hline $25-44$ & 11,228 & 26.4 & 15,137 & 35.4 & $9.0^{* * *}$ & $34.1 * * *$ & 3,862 & 9.1 & 4,527 & 10.6 & $1.5^{* * *}$ & $16.5^{* * *}$ \\
\hline $45-64$ & 7,537 & 18.4 & 9,519 & 23.2 & $4.8^{* * *}$ & $26.1^{* * *}$ & 3,676 & 9.0 & 4124 & 10.0 & $1.0^{* * *}$ & $11.1^{* * *}$ \\
\hline \multicolumn{13}{|l|}{ Female } \\
\hline $15-24$ & 871 & 4.1 & 1,041 & 4.9 & $0.8^{* * *}$ & $19.5^{* * *}$ & 267 & 1.2 & 294 & 1.4 & 0.2 & 16.7 \\
\hline $25-44$ & 4,824 & 11.4 & 6,162 & 14.5 & $3.1^{* * *}$ & $27.2^{* * *}$ & 2,434 & 5.8 & 2,642 & 6.2 & $0.4^{* * * *}$ & $6.9^{* * *}$ \\
\hline $45-64$ & 5,147 & 12.0 & 5,876 & 13.6 & $1.6^{* * *}$ & $13.3^{* * *}$ & 3,525 & 8.2 & 3,672 & 8.5 & 0.3 & 3.7 \\
\hline \multicolumn{13}{|l|}{ Race and Hispanic origin** } \\
\hline White, non-Hispanic & 27,056 & 13.9 & 33,450 & 17.5 & $3.6^{* * *}$ & $25.9^{* * *}$ & 12,894 & 6.4 & 14,167 & 7.0 & $0.6^{* * *}$ & $9.4^{* * *}$ \\
\hline Black, non-Hispanic & 2,741 & 6.6 & 4,374 & 10.3 & $3.7^{* * *}$ & $56.1^{* * *}$ & 1,060 & 2.6 & 1,392 & 3.3 & $0.7^{* * *}$ & $26.9^{* * *}$ \\
\hline Hispanic & 2,507 & 4.6 & 3,440 & 6.1 & $1.5^{* * *}$ & $32.6^{* * *}$ & 961 & 1.8 & 1,133 & 2.1 & $0.3^{* * * *}$ & $16.7^{* * *}$ \\
\hline Al/AN, non-Hispanic & 315 & 12.1 & 369 & 13.9 & 1.8 & 14.9 & 181 & 7.0 & 173 & 6.5 & -0.5 & -7.1 \\
\hline A/PI, non-Hispanic & 220 & 1.1 & 323 & 1.5 & $0.4^{* * *}$ & $36.4^{* * *}$ & 89 & 0.5 & 131 & 0.7 & 0.2 & 40 \\
\hline \multicolumn{13}{|c|}{ County urbanization level ${ }^{\dagger \dagger}$} \\
\hline Large central metro & 9,679 & 9.4 & 12,903 & 12.5 & $3.1^{* * *}$ & $33.0^{* * *}$ & 4,276 & 4.1 & 4,930 & 4.7 & $0.6^{* * *}$ & $14.6^{* * *}$ \\
\hline Large fringe metro & 8,683 & 11.2 & 11,993 & 15.4 & $4.2^{* * *}$ & $37.5^{* * *}$ & 3,444 & 4.2 & 4,209 & 5.2 & $1.0^{* * *}$ & $23.8^{* * *}$ \\
\hline Medium metro & 7,618 & 11.8 & 9,264 & 14.3 & $2.5^{* * * *}$ & $21.2^{* * *}$ & 3,664 & 5.6 & 3,988 & 6.0 & $0.4^{* * *}$ & $7.1^{* * *}$ \\
\hline Small metro & 2,729 & 9.9 & 3,224 & 11.7 & $1.8^{* * *}$ & $18.2^{* * *}$ & 1,404 & 5.0 & 1,471 & 5.2 & 0.2 & 4.0 \\
\hline Micropolitan (nonmetro) & 2,730 & 10.8 & 3,068 & 12.1 & $1.3^{* * *}$ & $12.0^{* * *}$ & 1,457 & 5.6 & 1,475 & 5.7 & 0.1 & 1.8 \\
\hline Noncore (nonmetro) & 1,652 & 9.6 & 1,797 & 10.5 & $0.9^{* * *}$ & $9.4^{* * *}$ & 1,036 & 5.9 & 1,014 & 5.7 & -0.2 & -3.4 \\
\hline \multicolumn{13}{|l|}{ Selected states $§ \S$} \\
\hline \multicolumn{13}{|c|}{ States with very good to excellent reporting $(n=25)$} \\
\hline Alaska & 86 & 11.0 & 94 & 12.5 & 1.5 & 13.6 & 57 & 7.4 & 51 & 6.8 & -0.6 & -8.1 \\
\hline Connecticut & 685 & 19.2 & 855 & 24.5 & $5.3^{* * *}$ & $27.6^{* * *}$ & 243 & 6.3 & 264 & 7.2 & 0.9 & 14.3 \\
\hline District of Columbia & 98 & 14.5 & 209 & 30.0 & $15.5^{* * *}$ & $106.9 * * *$ & 26 & 3.7 & 66 & 9.3 & $5.6^{* * *}$ & $151.4^{* * *}$ \\
\hline Georgia & 858 & 8.4 & 918 & 8.8 & 0.4 & 4.8 & 519 & 5.0 & 536 & 5.1 & 0.1 & 2.0 \\
\hline Illinois & 1,381 & 10.7 & 1,947 & 15.3 & $4.6^{* * *}$ & $43.0^{* * *}$ & 351 & 2.7 & 479 & 3.7 & $1.0^{* * * *}$ & $37.0^{* * *}$ \\
\hline lowa & 170 & 5.8 & 183 & 6.2 & 0.4 & 6.9 & 92 & 3.1 & 92 & 3.1 & 0.0 & 0.0 \\
\hline Maine & 238 & 19.3 & 301 & 25.2 & $5.9^{* * *}$ & $30.6^{* * *}$ & 124 & 9.6 & 154 & 12.5 & $2.9^{* * *}$ & $30.2^{* * *}$ \\
\hline Maryland & 1,087 & 17.7 & 1,821 & 29.7 & $12.0 * * *$ & $67.8^{* * *}$ & 534 & 8.7 & 812 & 13.1 & $4.4^{* * *}$ & $50.6^{* * *}$ \\
\hline Massachusetts & 1,550 & 23.3 & 1,990 & 29.7 & $6.4^{* * *}$ & $27.5^{* * *}$ & 298 & 4.3 & 351 & 4.9 & 0.6 & 14.0 \\
\hline Nevada & 419 & 13.8 & 408 & 13.3 & -0.5 & -3.6 & 298 & 9.8 & 275 & 8.9 & -0.9 & -9.2 \\
\hline New Hampshire & 380 & 31.3 & 437 & 35.8 & 4.5 & 14.4 & 80 & 5.7 & 89 & 6.5 & 0.8 & 14.0 \\
\hline
\end{tabular}

See table footnotes on the next page.

opioid and benzodiazepine pills, heroin, and cocaine, likely contributing to increases in overdose death rates involving other substances $(3,7,8)$.

The findings in this report are subject to at least five limitations. First, at autopsy, substances tested for, and circumstances under which tests are performed to determine which drugs are present, vary by time and jurisdiction, and improvements in toxicologic testing might account for some reported increases. Second, 17\% (2015) and 15\% (2016) of drug overdose death certificates did not include the specific types of drugs involved, 
TABLE 1. (Continued) Annual number and age-adjusted rate of drug overdose deaths* involving any opioid ${ }^{\dagger}$ and prescription opioids, ${ }^{\S, \uparrow}$ by sex, age, race and Hispanic origin, ${ }^{* *}$ urbanization level, ${ }^{\dagger \dagger}$ and selected states ${ }^{\S \S}$ - United States, 2015 and 2016

\begin{tabular}{|c|c|c|c|c|c|c|c|c|c|c|c|c|}
\hline \multirow[b]{3}{*}{ Decedent characteristic } & \multicolumn{6}{|c|}{ Opioids } & \multicolumn{6}{|c|}{ Prescription opioids } \\
\hline & \multicolumn{2}{|c|}{2015} & \multicolumn{2}{|c|}{2016} & \multicolumn{2}{|c|}{$\begin{array}{l}\text { Change from } \\
2015 \text { to } 2016^{\text {ๆी }}\end{array}$} & \multicolumn{2}{|c|}{2015} & \multicolumn{2}{|c|}{2016} & \multicolumn{2}{|c|}{$\begin{array}{l}\text { Change from } \\
2015 \text { to } 2016 \text { ๆๆ }\end{array}$} \\
\hline & No. & Rate & No. & Rate & $\begin{array}{l}\text { Absolute } \\
\text { rate change }\end{array}$ & $\begin{array}{l}\% \text { Change } \\
\text { in rate }\end{array}$ & No. & Rate & No. & Rate & $\begin{array}{c}\text { Absolute } \\
\text { rate change }\end{array}$ & $\begin{array}{c}\% \text { Change } \\
\text { in rate }\end{array}$ \\
\hline New Mexico & 351 & 17.9 & 349 & 17.5 & -0.4 & -2.2 & 189 & 9.6 & 186 & 9.2 & -0.4 & -4.2 \\
\hline New York & 2,166 & 10.8 & 3,009 & 15.1 & $4.3^{* * *}$ & $39.8^{* * *}$ & 895 & 4.4 & 1,100 & 5.4 & $1.0^{* * *}$ & $22.7^{* * *}$ \\
\hline North Carolina & 1,171 & 11.9 & 1,506 & 15.4 & $3.5^{* * *}$ & $29.4^{* * *}$ & 635 & 6.4 & 695 & 6.9 & 0.5 & 7.8 \\
\hline Ohio & 2,698 & 24.7 & 3,613 & 32.9 & $8.2^{* * *}$ & $33.2^{* * *}$ & 780 & 6.9 & 867 & 7.7 & $0.8^{* * *}$ & $11.6^{* * *}$ \\
\hline Oklahoma & 427 & 11.2 & 444 & 11.6 & 0.4 & 3.6 & 328 & 8.6 & 322 & 8.4 & -0.2 & -2.3 \\
\hline Oregon & 331 & 7.9 & 312 & 7.6 & -0.3 & -3.8 & 198 & 4.7 & 165 & 3.9 & -0.8 & -17.0 \\
\hline Rhode Island & 254 & 23.5 & 279 & 26.7 & 3.2 & 13.6 & 122 & 10.6 & 114 & 10.5 & -0.1 & -0.9 \\
\hline South Carolina & 554 & 11.4 & 628 & 13.1 & $1.7^{* * *}$ & $14.9 * * *$ & 361 & 7.3 & 381 & 7.8 & 0.5 & 6.8 \\
\hline Tennessee & 1,038 & 16.0 & 1,186 & 18.1 & $2.1^{* * *}$ & $13.1^{* * *}$ & 693 & 10.5 & 739 & 11.1 & 0.6 & 5.7 \\
\hline Utah & 448 & 15.9 & 466 & 16.4 & 0.5 & 3.1 & 385 & 13.7 & 349 & 12.5 & -1.2 & -8.8 \\
\hline Vermont & 79 & 13.4 & 101 & 18.4 & 5.0 & 37.3 & 32 & 5.3 & 35 & 5.9 & 0.6 & 11.3 \\
\hline Virginia & 820 & 9.9 & 1,130 & 13.5 & $3.6^{* * *}$ & $36.4^{* * *}$ & 322 & 3.8 & 400 & 4.7 & $0.9^{* * *}$ & $23.7^{* * *}$ \\
\hline Washington & 692 & 9.3 & 709 & 9.4 & 0.1 & 1.1 & 355 & 4.7 & 388 & 5.0 & 0.3 & 6.4 \\
\hline West Virginia & 629 & 36.0 & 733 & 43.4 & $7.4^{* * *}$ & $20.6^{* * *}$ & 380 & 21.2 & 340 & 19.7 & -1.5 & -7.1 \\
\hline \multicolumn{13}{|c|}{ States with good reporting $(n=7)$} \\
\hline Arizona & 671 & 10.2 & 769 & 11.4 & $1.2^{* * *}$ & $11.8^{* * *}$ & 362 & 5.5 & 380 & 5.6 & 0.1 & 1.8 \\
\hline Colorado & 495 & 8.7 & 536 & 9.5 & 0.8 & 9.2 & 288 & 5.1 & 258 & 4.5 & -0.6 & -11.8 \\
\hline Hawaii & 62 & 4.1 & 77 & 5.2 & 1.1 & 26.8 & 42 & 2.8 & 55 & 3.6 & 0.8 & 28.6 \\
\hline Minnesota & 338 & 6.2 & 396 & 7.4 & $1.2^{* * *}$ & $19.4^{* * *}$ & 177 & 3.2 & 195 & 3.6 & 0.4 & 12.5 \\
\hline Missouri & 692 & 11.7 & 914 & 15.9 & $4.2^{* * * *}$ & $35.9^{* * *}$ & 289 & 4.8 & 268 & 4.5 & -0.3 & -6.3 \\
\hline Texas & 1,287 & 4.7 & 1,375 & 4.9 & 0.2 & 4.3 & 590 & 2.1 & 617 & 2.2 & 0.1 & 4.8 \\
\hline Wisconsin & 622 & 11.2 & 866 & 15.8 & $4.6^{* * *}$ & $41.1^{* * *}$ & 300 & 5.2 & 382 & 6.7 & $1.5^{* * * *}$ & $28.8^{* * *}$ \\
\hline
\end{tabular}

Source: National Vital Statistics System, Mortality file.

Abbreviations: $\mathrm{A} / \mathrm{PI}=$ Asian/Pacific Islander; Al/AN = American Indian/Alaska Native.

* Deaths are classified using the International Classification of Diseases, Tenth Revision (ICD-10). Drug overdose deaths are identified using underlying cause-of-death codes X40-X44, X60-X64, X85, and Y10-Y14. Rates are age-adjusted using the direct method and the 2000 U.S. standard population, except for age-specific crude rates. All rates are per 100,000 population. Cells with $\leq 9$ deaths are not reported. Rates based on $<20$ deaths are not considered reliable and not reported.

${ }^{\dagger}$ Drug overdose deaths, as defined, that have opium (T40.0), heroin (T40.1), natural and semisynthetic opioids (T40.2), methadone (T40.3), synthetic opioids other than methadone (T40.4), or other and unspecified narcotics (T40.6) as a contributing cause.

$\S$ Drug overdose deaths, as defined, that have natural and semisynthetic opioids (T40.2) or methadone (T40.3) as a contributing cause.

" Categories of deaths are not exclusive because deaths may involve more than one drug. Summing of categories will result in greater than the total number of deaths in a year.

** Data for Hispanic origin should be interpreted with caution; studies comparing Hispanic origin on death certificates and on census surveys have shown inconsistent reporting on Hispanic ethnicity. Potential race misclassification might lead to underestimates for certain categories, primarily Al/AN non-Hispanic and A/PI nonHispanic decedents. https://www.cdc.gov/nchs/data/series/sr_02/sr02_172.pdf.

${ }^{\dagger+}$ By 2013 urbanization classification. https://www.cdc.gov/nchs/data_access/urban_rural.htm.

$\S \S$ Analyses were limited to states meeting the following criteria: for states with very good to excellent reporting, $\geq 90 \%$ of drug overdose deaths mention at least one specific drug in 2015, with the change in drug overdose deaths mentioning at least one specific drug differing by no more than 10 percentage points from 2015 to 2016 . States with good reporting had $80 \%$ to $<90 \%$ of drug overdose deaths mention of at least one specific drug in 2015 , with the change in the percentage of drug overdose deaths mentioning at least one specific drug differing by $<10$ percentage points from 2015 to 2016 . States included also were required to have stable rate estimates, based on $\geq 20$ deaths, in at least two drug categories (i.e., opioids, prescription opioids, synthetic opioids other than methadone, heroin, cocaine, and psychostimulants with abuse potential). South Dakota was the only state with good or excellent reporting in both years, but with an improvement $>10$ percentage points in drug specificity.

१ी Absolute rate change is the difference between 2015 and 2016 rates. Percent change is the absolute rate change divided by the 2015 rate, multiplied by 100. Nonoverlapping confidence intervals based on the gamma method were used if the number of deaths was $<100$ in 2015 or 2016, and z-tests were used if the number of deaths was $\geq 100$ in both 2015 and 2016. Note that the method of comparing confidence intervals is a conservative method for statistical significance; caution should be observed when interpreting a nonsignificant difference when the lower and upper limits being compared overlap only slightly. Confidence intervals of 2015 and 2016 rates of prescription opioid deaths for Asian/Pacific Islanders overlapped only slightly: $(0.37,0.57),(0.56,0.80)$.

*** Statistically significant at 0.05 level.

and the percentage of drug overdose death certificates with at least one drug specified varied widely by state, ranging from $52.5 \%$ to $99.3 \%$ in 2016 . This variation limits rate comparisons between states. Third, because heroin and morphine are metabolized similarly (9), some heroin deaths might have been misclassified as morphine deaths, resulting in underreporting of heroin deaths. Fourth, potential race misclassification might lead to underestimates for certain categories, primarily for American Indian/Alaska Natives and Asian/Pacific Islanders. 9999 Finally, state-specific analyses are restricted to 31 states and DC, limiting generalizability.

S999 https://www.cdc.gov/nchs/data/series/sr_02/sr02_172.pdf. 
TABLE 2. Annual number and age-adjusted rate of drug overdose deaths* involving heroin ${ }^{\dagger}$ and synthetic opioids other than methadone, $\$, \eta$ by sex, age, race and Hispanic origin, ${ }^{* *}$ urbanization level, ${ }^{\dagger \dagger}$ and selected states ${ }^{\S \S}$ — United States, 2015 and 2016

\begin{tabular}{|c|c|c|c|c|c|c|c|c|c|c|c|c|}
\hline \multirow[b]{3}{*}{ Decedent characteristic } & \multicolumn{6}{|c|}{ Heroin } & \multicolumn{6}{|c|}{ Synthetic opioids other than methadone } \\
\hline & \multicolumn{2}{|c|}{2015} & \multicolumn{2}{|c|}{2016} & \multicolumn{2}{|c|}{$\begin{array}{l}\text { Change from } \\
2015 \text { to } 2016 \text { ๆึ }\end{array}$} & \multicolumn{2}{|c|}{2015} & \multicolumn{2}{|c|}{2016} & \multicolumn{2}{|c|}{$\begin{array}{l}\text { Change from } \\
2015 \text { to } 2016 \text { ๆๆ }\end{array}$} \\
\hline & No. & Rate & No. & Rate & $\begin{array}{c}\text { Absolute } \\
\text { rate change }\end{array}$ & $\begin{array}{l}\% \text { Change } \\
\text { in rate }\end{array}$ & No. & Rate & No. & Rate & $\begin{array}{c}\text { Absolute } \\
\text { rate change }\end{array}$ & $\begin{array}{c}\% \text { Change } \\
\text { in rate }\end{array}$ \\
\hline All & 12,989 & 4.1 & 15,469 & 4.9 & $0.8^{* * *}$ & $19.5^{* * *}$ & 9,580 & 3.1 & 19,413 & 6.2 & $3.1^{* * *}$ & $100.0 * * *$ \\
\hline \multicolumn{13}{|l|}{ Sex } \\
\hline Male & 9,881 & 6.3 & 11,752 & 7.5 & $1.2^{* * *}$ & $19.0^{* * *}$ & 6,560 & 4.2 & 13,835 & 8.9 & $4.7^{* * *}$ & $111.9^{* * *}$ \\
\hline Female & 3,108 & 2.0 & 3,717 & 2.4 & $0.4^{* * *}$ & $20.0^{* * *}$ & 3,020 & 1.9 & 5,578 & 3.5 & $1.6^{* * *}$ & $84.2^{* * *}$ \\
\hline \multicolumn{13}{|l|}{ Age group (yrs) } \\
\hline $0-14$ & - & - & - & - & - & - & 14 & - & 18 & $-{ }^{\dagger+\dagger}$ & - & - \\
\hline $15-24$ & 1,649 & 3.8 & 1,728 & 4.0 & 0.2 & 5.3 & 999 & 2.3 & 1,958 & 4.5 & $2.2^{* * *}$ & $95.7^{* * *}$ \\
\hline $25-34$ & 4,292 & 9.7 & 5,051 & 11.3 & $1.6^{* * *}$ & $16.5^{* * *}$ & 2,896 & 6.6 & 6,094 & 13.6 & $7.0^{* * *}$ & $106.1^{* * *}$ \\
\hline $35-44$ & 3,012 & 7.4 & 3,625 & 9.0 & $1.6^{* * *}$ & $21.6^{* * *}$ & 2,289 & 5.6 & 4,825 & 11.9 & $6.3^{* * *}$ & $112.5^{* * * *}$ \\
\hline $45-54$ & 2,439 & 5.6 & 3,009 & 7.0 & $1.4^{* * *}$ & $25.0 * * *$ & 1,982 & 4.6 & 3,872 & 9.1 & $4.5^{* * *}$ & $97.8^{* * *}$ \\
\hline $55-64$ & 1,407 & 3.4 & 1,777 & 4.3 & $0.9^{* * *}$ & $26.5^{* * *}$ & 1,167 & 2.9 & 2,238 & 5.4 & $2.5^{* * *}$ & $86.2^{* * *}$ \\
\hline$\geq 65$ & 184 & 0.4 & 275 & 0.6 & $0.2^{* * *}$ & $50.0^{* * *}$ & 232 & 0.5 & 405 & 0.8 & $0.3^{* * *}$ & $60.0^{* * *}$ \\
\hline \multicolumn{13}{|l|}{ Sex and age group (yrs) } \\
\hline \multicolumn{13}{|l|}{ Male } \\
\hline $15-24$ & 1,172 & 5.2 & 1,275 & 5.7 & $0.5^{* * *}$ & $9.6^{* * *}$ & 718 & 3.2 & 1,434 & 6.4 & $3.2^{* * *}$ & $100.0^{* * * *}$ \\
\hline $25-44$ & 5,602 & 13.2 & 6,643 & 15.5 & $2.3^{* * *}$ & $17.4^{* * *}$ & 3,764 & 8.9 & 8,029 & 18.8 & $9.9^{* * *}$ & $111.2^{* * *}$ \\
\hline $45-64$ & 2,953 & 7.2 & 3,599 & 8.8 & $1.6^{* * *}$ & $22.2^{* * *}$ & 1,948 & 4.8 & 4,116 & 10.0 & $5.2^{* * *}$ & $108.3^{* * *}$ \\
\hline \multicolumn{13}{|l|}{ Female } \\
\hline $15-24$ & 477 & 2.2 & 453 & 2.1 & -0.1 & -4.5 & 281 & 1.3 & 524 & 2.5 & $1.2^{* * *}$ & $92.3^{* * *}$ \\
\hline $25-44$ & 1,702 & 4.0 & 2,033 & 4.8 & $0.8^{* * *}$ & $20.0^{* * *}$ & 1,421 & 3.4 & 2,890 & 6.8 & $3.4^{* * *}$ & $100.0^{* * *}$ \\
\hline $45-64$ & 893 & 2.1 & 1,187 & 2.8 & $0.7^{* * *}$ & $33.3^{* * *}$ & 1,201 & 2.8 & 1,994 & 4.6 & $1.8^{* * *}$ & $64.3^{* * *}$ \\
\hline \multicolumn{13}{|c|}{ Race and Hispanic origin ${ }^{* *}$} \\
\hline White, non-Hispanic & 10,050 & 5.4 & 11,631 & 6.3 & $0.9^{* * *}$ & $16.7^{* * *}$ & 7,995 & 4.2 & 15,143 & 8.2 & $4.0^{* * *}$ & $95.2^{* * *}$ \\
\hline Black, non-Hispanic & 1,310 & 3.1 & 1,899 & 4.5 & $1.4^{* * *}$ & $45.2^{* * *}$ & 883 & 2.1 & 2,391 & 5.6 & $3.5^{* * *}$ & $166.7^{* * *}$ \\
\hline Hispanic & 1,299 & 2.3 & 1,555 & 2.8 & $0.5^{* * *}$ & $21.7^{* * *}$ & 524 & 0.9 & 1,505 & 2.7 & $1.8^{* * *}$ & $200.0^{* * *}$ \\
\hline Al/AN, non-Hispanic & 117 & 4.4 & 131 & 5.0 & 0.6 & 13.6 & 51 & 2.0 & 113 & 4.1 & $2.1^{* * *}$ & $105.0^{* * *}$ \\
\hline A/PI, non-Hispanic & 98 & 0.5 & 102 & 0.5 & 0.0 & 0.0 & 51 & 0.2 & 134 & 0.6 & $0.4^{* * *}$ & $200.0^{* * *}$ \\
\hline \multicolumn{13}{|c|}{ County urbanization level ${ }^{\dagger \dagger}$} \\
\hline Large central metro & 4,496 & 4.4 & 5,507 & 5.3 & $0.9 * * *$ & $20.5^{* * *}$ & 2,509 & 2.4 & 6,009 & 5.8 & $3.4^{* * *}$ & $141.7^{* * *}$ \\
\hline Large fringe metro & 3,778 & 5.0 & 4,623 & 6.1 & $1.1^{* * *}$ & $22.0^{* * *}$ & 2,947 & 3.9 & 6,264 & 8.2 & $4.3^{* * *}$ & $110.3^{* * *}$ \\
\hline Medium metro & 2,736 & 4.3 & 3,077 & 4.9 & $0.6^{* * *}$ & $14.0^{* * *}$ & 2,255 & 3.5 & 3,978 & 6.3 & $2.8^{* * *}$ & $80.0^{* * *}$ \\
\hline Small metro & 868 & 3.2 & 990 & 3.7 & $0.5^{* * *}$ & $15.6^{* * *}$ & 686 & 2.5 & 1,270 & 4.7 & $2.2^{* * *}$ & $88.0^{* * *}$ \\
\hline Micropolitan (nonmetro) & 778 & 3.2 & 860 & 3.6 & $0.4^{* * *}$ & $12.5^{* * *}$ & 753 & 3.0 & 1,228 & 5.0 & $2.0^{* * *}$ & $66.7^{* * *}$ \\
\hline Noncore (nonmetro) & 333 & 2.1 & 412 & 2.6 & $0.5^{* * *}$ & $23.8^{* * *}$ & 430 & 2.6 & 664 & 4.1 & $1.5^{* * *}$ & $57.7^{* * *}$ \\
\hline \multicolumn{13}{|l|}{ Selected states ${ }^{\S \S}$} \\
\hline \multicolumn{13}{|c|}{ States with very good to excellent reporting $(n=25)$} \\
\hline Alaska & 37 & 4.7 & 49 & 6.5 & 1.8 & 38.3 & 14 & - $^{\dagger+\dagger}$ & - & - & $-{ }^{++\dagger}$ & $-{ }^{\dagger+\dagger}$ \\
\hline Connecticut & 390 & 11.3 & 450 & 13.1 & $1.8^{* * *}$ & $15.9^{* * *}$ & 211 & 6.1 & 500 & 14.8 & $8.7^{* * *}$ & $142.6^{* * *}$ \\
\hline District of Columbia & 67 & 9.9 & 122 & 17.3 & $7.4^{* * *}$ & $74.7^{* * *}$ & 26 & 3.9 & 129 & 19.2 & $15.3^{* * *}$ & $392.3^{* * *}$ \\
\hline Georgia & 222 & 2.2 & 226 & 2.2 & 0.0 & 0.0 & 284 & 2.8 & 277 & 2.7 & -0.1 & -3.6 \\
\hline Illinois & 844 & 6.7 & 1,040 & 8.2 & $1.5^{* * *}$ & $22.4^{* * *}$ & 278 & 2.2 & 907 & 7.2 & $5.0^{* * *}$ & $227.3^{* * *}$ \\
\hline lowa & 45 & 1.6 & 47 & 1.7 & 0.1 & 6.2 & 44 & 1.5 & 58 & 2.0 & 0.5 & 33.3 \\
\hline Maine & 52 & 4.5 & 55 & 4.7 & 0.2 & 4.4 & 116 & 9.9 & 199 & 17.3 & $7.4^{* * *}$ & $74.7^{* * *}$ \\
\hline Maryland & 405 & 6.6 & 650 & 10.7 & $4.1^{* * *}$ & $62.1 * * *$ & 357 & 5.8 & 1,091 & 17.8 & $12.0^{* * *}$ & $206.9^{* * *}$ \\
\hline Massachusetts & 634 & 9.6 & 630 & 9.5 & -0.1 & -1.0 & 949 & 14.4 & 1,550 & 23.5 & $9.1^{* * *}$ & $63.2^{* * *}$ \\
\hline Nevada & 82 & 2.7 & 86 & 2.9 & 0.2 & 7.4 & 32 & 1.1 & 53 & 1.7 & 0.6 & 54.5 \\
\hline New Hampshire & 78 & 6.5 & 34 & 2.8 & $-3.7^{* * *}$ & $-56.9 * * *$ & 285 & 24.1 & 363 & 30.3 & $6.2^{* * *}$ & $25.7^{* * *}$ \\
\hline
\end{tabular}

See table footnotes on the next page.

The ongoing and worsening drug overdose epidemic requires immediate attention and action. Faster access to data collected is needed to understand emerging threats in local communities and to tailor response activities. CDC's Enhanced State Opioid Overdose Surveillance program funds 32 states and
DC for more timely and comprehensive nonfatal and fatal overdose data, including funding for improved comprehensive toxicologic testing to identify emerging drug threats in opioid-involved fatal overdoses. ${ }^{* * * * *}$ Syndromic surveillance

***** https://www.cdc.gov/drugoverdose/foa/state-opioid-mm.html. 
TABLE 2. (Continued) Annual number and age-adjusted rate of drug overdose deaths* involving heroin ${ }^{\dagger}$ and synthetic opioids other than methadone, ${ }^{\S, \uparrow}$ by sex, age, race and Hispanic origin, ${ }^{* *}$ urbanization level, ${ }^{\dagger \dagger}$ and selected states ${ }^{\S \S}$ - United States, 2015 and 2016

\begin{tabular}{|c|c|c|c|c|c|c|c|c|c|c|c|c|}
\hline \multirow[b]{3}{*}{ Decedent characteristic } & \multicolumn{6}{|c|}{ Heroin } & \multicolumn{6}{|c|}{ Synthetic opioids other than methadone } \\
\hline & \multicolumn{2}{|c|}{2015} & \multicolumn{2}{|c|}{2016} & \multicolumn{2}{|c|}{$\begin{array}{l}\text { Change from } \\
2015 \text { to } 2016 \text { ๆๆ }\end{array}$} & \multicolumn{2}{|c|}{2015} & \multicolumn{2}{|c|}{2016} & \multicolumn{2}{|c|}{$\begin{array}{l}\text { Change from } \\
2015 \text { to } 2016 \text { ๆๆ }\end{array}$} \\
\hline & No. & Rate & No. & Rate & $\begin{array}{c}\text { Absolute } \\
\text { rate change }\end{array}$ & $\begin{array}{l}\% \text { Change } \\
\text { in rate }\end{array}$ & No. & Rate & No. & Rate & $\begin{array}{l}\text { Absolute } \\
\text { rate change }\end{array}$ & $\begin{array}{l}\% \text { Change } \\
\text { in rate }\end{array}$ \\
\hline New Mexico & 156 & 8.1 & 161 & 8.2 & 0.1 & 1.2 & 42 & 2.1 & 78 & 4.0 & $1.9^{* * *}$ & $90.5^{* * *}$ \\
\hline New York & 1,058 & 5.4 & 1,307 & 6.5 & $1.1^{* * *}$ & $20.4^{* * *}$ & 668 & 3.3 & 1,641 & 8.3 & $5.0 * * *$ & $151.5^{* * *}$ \\
\hline North Carolina & 393 & 4.1 & 544 & 5.7 & $1.6^{* * *}$ & $39.0^{* * *}$ & 300 & 3.1 & 601 & 6.2 & $3.1^{* * *}$ & $100.0^{* * *}$ \\
\hline Ohio & 1,444 & 13.3 & 1,478 & 13.5 & 0.2 & 1.5 & 1,234 & 11.4 & 2,296 & 21.1 & $9.7^{* * *}$ & $85.1^{* * *}$ \\
\hline Oklahoma & 36 & 1.0 & 53 & 1.4 & 0.4 & 40.0 & 93 & 2.4 & 98 & 2.5 & 0.1 & 4.2 \\
\hline Oregon & 102 & 2.5 & 114 & 2.9 & 0.4 & 16.0 & 34 & 0.9 & 43 & 1.1 & 0.2 & 22.2 \\
\hline Rhode Island & 45 & 4.3 & 25 & 2.5 & -1.8 & -41.9 & 137 & 13.2 & 182 & 17.8 & $4.6^{* * *}$ & $34.8^{* * *}$ \\
\hline South Carolina & 100 & 2.2 & 115 & 2.5 & 0.3 & 13.6 & 161 & 3.3 & 237 & 5.0 & $1.7^{* * *}$ & $51.5^{* * *}$ \\
\hline Tennessee & 205 & 3.3 & 260 & 4.1 & $0.8^{* * *}$ & $24.2^{* * *}$ & 251 & 4.0 & 395 & 6.2 & $2.2^{* * *}$ & $55.0 * * *$ \\
\hline Utah & 127 & 4.3 & 166 & 5.6 & $1.3^{* * *}$ & $30.2^{* * *}$ & 62 & 2.3 & 72 & 2.5 & 0.2 & 8.7 \\
\hline Vermont & 33 & 5.8 & 45 & 8.7 & 2.9 & 50.0 & 33 & 5.6 & 53 & 10.1 & 4.5 & 80.4 \\
\hline Virginia & 353 & 4.3 & 450 & 5.5 & $1.2^{* * *}$ & $27.9 * * *$ & 270 & 3.3 & 648 & 7.9 & $4.6^{* * *}$ & $139.4^{* * *}$ \\
\hline Washington & 303 & 4.2 & 283 & 3.9 & -0.3 & -7.1 & 65 & 0.9 & 93 & 1.3 & 0.4 & 44.4 \\
\hline West Virginia & 194 & 11.8 & 235 & 14.9 & $3.1^{* * *}$ & $26.3^{* * *}$ & 217 & 12.7 & 435 & 26.3 & $13.6^{* * *}$ & $107.1^{* * *}$ \\
\hline \multicolumn{13}{|c|}{ States with good reporting $(n=7)$} \\
\hline Arizona & 247 & 3.8 & 299 & 4.5 & $0.7^{* * *}$ & $18.4^{* * *}$ & 72 & 1.1 & 123 & 1.8 & $0.7^{* * *}$ & $63.6^{* * *}$ \\
\hline Colorado & 159 & 2.8 & 234 & 4.2 & $1.4^{* * *}$ & $50.0^{* * *}$ & 64 & 1.2 & 72 & 1.3 & 0.1 & 8.3 \\
\hline Hawaii & 15 & - & 20 & 1.4 & — & _t+† & 13 & - & - & - & - & —t+† \\
\hline Minnesota & 115 & 2.2 & 149 & 2.8 & 0.6 & 27.3 & 55 & 1.0 & 99 & 1.9 & $0.9^{* * *}$ & $90.0^{* * *}$ \\
\hline Missouri & 303 & 5.3 & 380 & 6.7 & $1.4^{* * *}$ & $26.4^{* * *}$ & 183 & 3.1 & 441 & 7.8 & $4.7^{* * *}$ & $151.6^{* * *}$ \\
\hline Texas & 523 & 1.9 & 530 & 1.9 & 0.0 & 0.0 & 186 & 0.7 & 250 & 0.9 & $0.2^{* * *}$ & $28.6^{* * *}$ \\
\hline Wisconsin & 287 & 5.3 & 389 & 7.3 & $2.0^{* * *}$ & $37.7^{* * *}$ & 112 & 2.1 & 288 & 5.3 & $3.2^{* * *}$ & $152.4^{* * *}$ \\
\hline
\end{tabular}

Source: National Vital Statistics System, Mortality file.

Abbreviations: $\mathrm{A} / \mathrm{PI}=$ Asian/Pacific Islander; Al/AN = American Indian/Alaska Native.

* Deaths are classified using the International Classification of Diseases, Tenth Revision (ICD-10). Drug overdose deaths are identified using underlying cause-of-death codes X40-X44, X60-X64, X85, and Y10-Y14. Rates are age-adjusted using the direct method and the 2000 U.S. standard population, except for age-specific crude rates. All rates are per 100,000 population.

${ }^{\dagger}$ Drug overdose deaths, as defined, that have heroin (T40.1) as a contributing cause.

§ Drug overdose deaths, as defined, that have synthetic opioids other than methadone (T40.4) as a contributing cause.

" Categories of deaths are not exclusive because deaths may involve more than one drug. Summing of categories will result in greater than the total number of deaths in a year.

** Data for Hispanic origin should be interpreted with caution; studies comparing Hispanic origin on death certificates and on census surveys have shown inconsistent reporting on Hispanic ethnicity. Potential race misclassification might lead to underestimates for certain categories, primarily Al/AN non-Hispanic and A/PI nonHispanic decedents. https://www.cdc.gov/nchs/data/series/sr_02/sr02_172.pdf.

${ }^{+\dagger}$ By 2013 urbanization classification. https://www.cdc.gov/nchs/data_access/urban_rural.htm.

$\S$ Analyses were limited to states meeting the following criteria: For states with very good to excellent reporting, $\geq 90 \%$ of drug overdose deaths mention at least one specific drug in 2015, with the change in drug overdose deaths mentioning at least one specific drug differing by <10 percentage points from 2015 to 2016 . States with good reporting had $80 \%$ to $<90 \%$ of drug overdose deaths mention of at least one specific drug in 2015 , with the change in the percentage of drug overdose deaths mentioning at least one specific drug differing by $<10$ percentage points from 2015 to 2016 . States included also were required to have stable rate estimates, based on $\geq 20$ deaths, in at least two drug categories (i.e., opioids, prescription opioids, synthetic opioids other than methadone, heroin, cocaine, and psychostimulants with abuse potential). South Dakota was the only state with good or excellent reporting in both years, but with an improvement $>10$ percentage points in drug specificity.

१ी Absolute rate change is the difference between 2015 and 2016 rates. Percent change is the absolute rate change divided by the 2015 rate, multiplied by 100. Nonoverlapping confidence intervals based on the gamma method were used if the number of deaths was $<100$ in 2015 or 2016, and z-tests were used if the number of deaths was $\geq 100$ in both 2015 and 2016.

*** Statistically significant at 0.05 level.

t+t Cells with $\leq 9$ deaths are not reported. Rates based on $<20$ deaths are not considered reliable and not reported.

data allow communities to identify overdoses quickly (10). The State Unintentional Drug Overdose Reporting System provides improved collection of toxicology data to identify specific drugs involved (G), information gathered from death scene investigations, and risk factors associated with fatal overdoses. Given the continuing threat from prescription opioids and the evolving threat from illicit opioids and other substances, a multifaceted prevention approach is required.
Efforts to ensure safe prescribing practices ${ }^{\dagger \dagger \dagger \dagger \dagger}$ are enhanced by access to nonopioid and nonpharmacologic treatments for pain. Other important efforts include increasing naloxone availability, expanding access to medication-assisted treatment, and maximizing the ability of health systems to link persons to treatment and harm reduction services (10). CDC supports

\footnotetext{
$\overline{\dagger \dagger \dagger \dagger \dagger}$ https://www.cdc.gov/drugoverdose/prescribing/guideline.html.
} 
TABLE 3. Annual number and age-adjusted rate of drug overdose deaths* involving cocaine ${ }^{\dagger}$ and psychostimulants with abuse potential,,$₫$ by sex, age, race and Hispanic origin, ${ }^{* *}$ urbanization level, ${ }^{\dagger \dagger}$ and selected states ${ }^{\S \S}$ — United States, 2015 and 2016

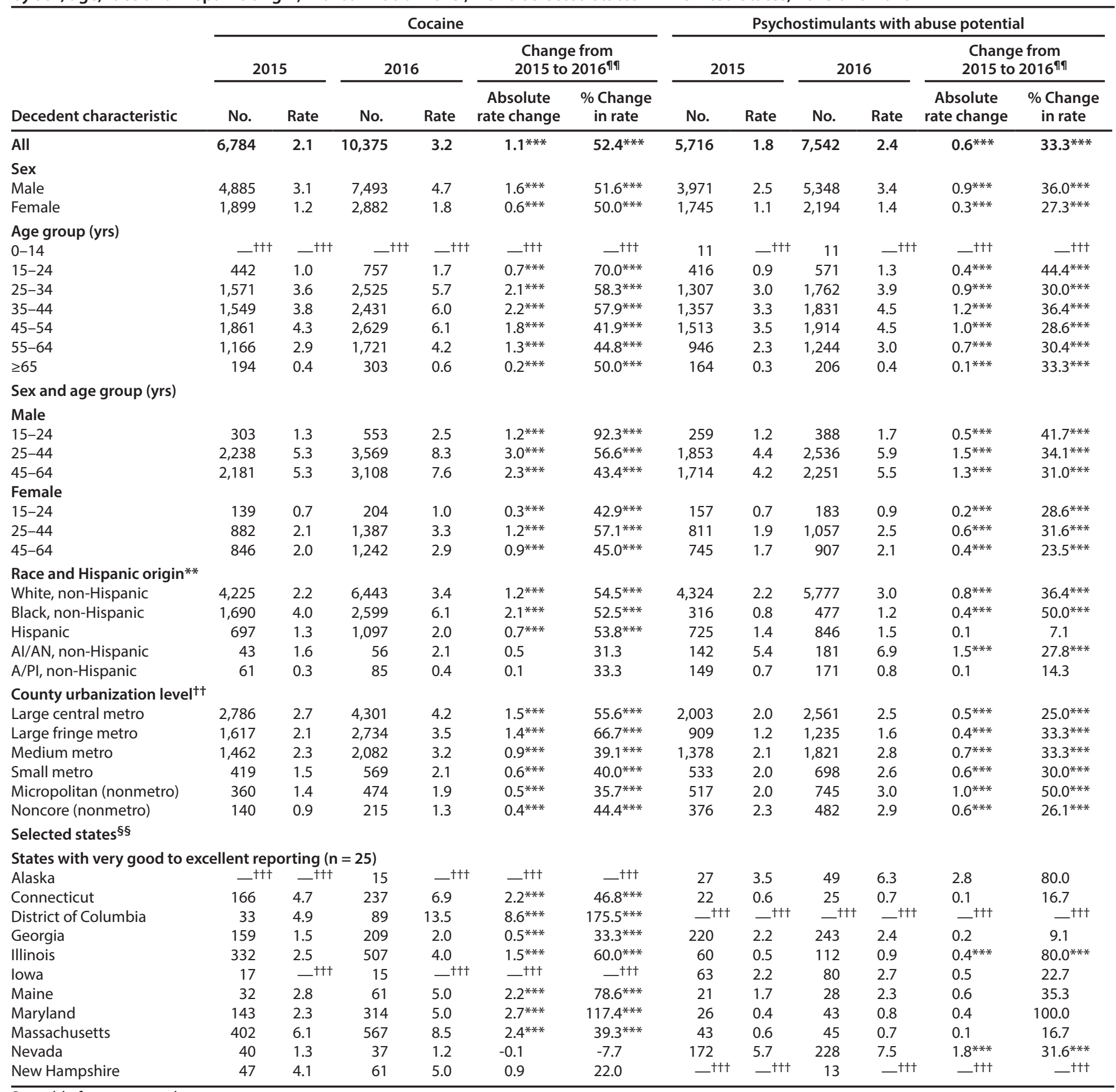

See table footnotes on the next page.

many of these efforts through the Prevention for States and

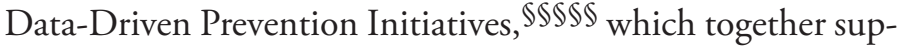
port opioid overdose prevention efforts in 42 states and DC.

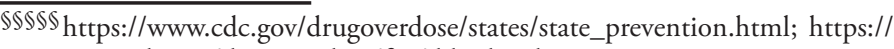
www.cdc.gov/drugoverdose/foa/ddpi.html.
Collaboration with law enforcement, first responders, and harm reduction partners is also important to understanding local variations in drug supply and lethality and to implementing a multisectoral prevention approach. 
TABLE 3. (Continued) Annual number and age-adjusted rate of drug overdose deaths* involving cocaine ${ }^{\dagger}$ and psychostimulants with abuse potential, ${ }^{\S, \uparrow}$ by sex, age, race and Hispanic origin, ${ }^{* *}$ urbanization level, ${ }^{\dagger \dagger}$ and selected states ${ }^{\S \S}$ — United States, 2015 and 2016

\begin{tabular}{|c|c|c|c|c|c|c|c|c|c|c|c|c|}
\hline \multirow[b]{3}{*}{ Decedent characteristic } & \multicolumn{6}{|c|}{ Cocaine } & \multicolumn{6}{|c|}{ Psychostimulants with abuse potential } \\
\hline & \multicolumn{2}{|c|}{2015} & \multicolumn{2}{|c|}{2016} & \multicolumn{2}{|c|}{$\begin{array}{l}\text { Change from } \\
2015 \text { to } 2016^{\text {ๆๆ }}\end{array}$} & \multicolumn{2}{|c|}{2015} & \multicolumn{2}{|c|}{2016} & \multicolumn{2}{|c|}{$\begin{array}{l}\text { Change from } \\
2015 \text { to } 2016 \text { ๆๆ }\end{array}$} \\
\hline & No. & Rate & No. & Rate & $\begin{array}{c}\text { Absolute } \\
\text { rate change }\end{array}$ & $\begin{array}{l}\% \text { Change } \\
\text { in rate }\end{array}$ & No. & Rate & No. & Rate & $\begin{array}{c}\text { Absolute } \\
\text { rate change }\end{array}$ & $\begin{array}{c}\% \text { Change } \\
\text { in rate }\end{array}$ \\
\hline New Mexico & 51 & 2.6 & 58 & 3.0 & 0.4 & 15.4 & 119 & 6.1 & 135 & 7.1 & 1.0 & 16.4 \\
\hline New York & 634 & 3.1 & 991 & 4.9 & $1.8^{* * *}$ & $58.1^{* * *}$ & 80 & 0.4 & 150 & 0.8 & $0.4^{* * *}$ & $100.0^{* * *}$ \\
\hline North Carolina & 314 & 3.2 & 500 & 5.1 & $1.9^{* * *}$ & $59.4^{* * *}$ & 67 & 0.7 & 115 & 1.2 & $0.5^{* * *}$ & $71.4^{* * *}$ \\
\hline Ohio & 698 & 6.3 & 1,124 & 10.1 & $3.8^{* * *}$ & $60.3^{* * *}$ & 105 & 1.0 & 243 & 2.3 & $1.3^{* * *}$ & $130.0^{* * *}$ \\
\hline Oklahoma & 29 & 0.7 & 31 & 0.8 & 0.1 & 14.3 & 199 & 5.3 & 263 & 7.1 & $1.8^{* * *}$ & $34.0^{* * *}$ \\
\hline Oregon & 22 & 0.6 & 26 & 0.7 & 0.1 & 16.7 & 124 & 3.1 & 150 & 3.6 & 0.5 & 16.1 \\
\hline Rhode Island & 87 & 8.3 & 112 & 10.7 & 2.4 & 28.9 & 11 & —十+十 & 10 & —†† & —†+ & —十+† \\
\hline South Carolina & 116 & 2.4 & 143 & 3.0 & 0.6 & 25.0 & 87 & 1.9 & 125 & 2.7 & 0.8 & 42.1 \\
\hline Tennessee & 202 & 3.0 & 249 & 3.8 & $0.8^{* * *}$ & $26.7^{* * *}$ & 113 & 1.8 & 186 & 2.9 & $1.1^{* * *}$ & $61.1^{* * *}$ \\
\hline Utah & 44 & 1.5 & 48 & 1.7 & 0.2 & 13.3 & 147 & 5.2 & 143 & 5.1 & -0.1 & -1.9 \\
\hline Vermont & 14 & _t†十 & 21 & 4.0 & —†† & — & —†t+ & —十+† & —†+ & —†+ & —†t+ & —t+† \\
\hline Virginia & 168 & 2.0 & 254 & 3.0 & $1.0^{* * *}$ & $50.0^{* * *}$ & 55 & 0.7 & 76 & 0.9 & 0.2 & 28.6 \\
\hline Washington & 85 & 1.1 & 90 & 1.2 & 0.1 & 9.1 & 304 & 4.2 & 326 & 4.4 & 0.2 & 4.8 \\
\hline West Virginia & 94 & 5.6 & 143 & 8.5 & $2.9^{* * *}$ & $51.8^{* * *}$ & 65 & 3.9 & 117 & 7.0 & $3.1 * * *$ & $79.5^{* * *}$ \\
\hline \multicolumn{13}{|c|}{ States with good reporting $(n=7)$} \\
\hline Arizona & 62 & 0.9 & 82 & 1.2 & 0.3 & 33.3 & 333 & 5.1 & 454 & 6.7 & $1.6^{* * *}$ & $31.4^{* * *}$ \\
\hline Colorado & 60 & 1.0 & 106 & 1.9 & $0.9^{* * *}$ & $90.0^{* * *}$ & 140 & 2.6 & 200 & 3.6 & $1.0^{* * *}$ & $38.5^{* * *}$ \\
\hline Hawaii & —†† & —†† & —†† & —十†t & —†† & —†† & 87 & 5.9 & 102 & 6.8 & 0.9 & 15.3 \\
\hline Minnesota & 42 & 0.7 & 43 & 0.8 & 0.1 & 14.3 & 82 & 1.5 & 140 & 2.6 & $1.1^{* * *}$ & $73.3^{* * *}$ \\
\hline Missouri & 77 & 1.3 & 103 & 1.8 & 0.5 & 38.5 & 133 & 2.4 & 185 & 3.3 & $0.9 * * *$ & $37.5^{* * *}$ \\
\hline Texas & 470 & 1.7 & 584 & 2.1 & $0.4^{* * *}$ & $23.5^{* * *}$ & 454 & 1.7 & 577 & 2.1 & $0.4^{* * *}$ & $23.5^{* * *}$ \\
\hline Wisconsin & 115 & 2.0 & 147 & 2.6 & $0.6^{* * * *}$ & $30.0^{* * *}$ & 38 & 0.7 & 76 & 1.4 & $0.7^{* * *}$ & $100.0^{* * *}$ \\
\hline
\end{tabular}

Source: National Vital Statistics System, Mortality file.

Abbreviations: $\mathrm{A} / \mathrm{PI}=$ Asian/Pacific Islander; Al/AN = American Indian/Alaska Native.

* Deaths are classified using the International Classification of Diseases, Tenth Revision (ICD-10). Drug overdose deaths are identified using underlying cause-of-death codes X40-X44, X60-X64, X85, and Y10-Y14. Rates are age-adjusted using the direct method and the 2000 U.S. standard population, except for age-specific crude rates. All rates are per 100,000 population.

† Drug overdose deaths, as defined, that have cocaine (T40.5) as a contributing cause.

$\S$ Drug overdose deaths, as defined, that have psychostimulants with abuse potential (T43.6) as a contributing cause.

" Categories of deaths are not exclusive because deaths may involve more than one drug. Summing of categories will result in greater than the total number of deaths in a year.

** Data for Hispanic origin should be interpreted with caution; studies comparing Hispanic origin on death certificates and on census surveys have shown inconsistent reporting on Hispanic ethnicity. Potential race misclassification might lead to underestimates for certain categories, primarily Al/AN non-Hispanic and A/PI nonHispanic decedents. https://www.cdc.gov/nchs/data/series/sr_02/sr02_172.pdf.

t† By 2013 urbanization classification. https://www.cdc.gov/nchs/data_access/urban_rural.htm.

$\S$ Analyses were limited to states meeting the following criteria: For states with very good to excellent reporting, $\geq 90 \%$ of drug overdose deaths mention at least one specific drug in 2015, with the change in drug overdose deaths mentioning at least one specific drug differing by $<10$ percentage points from 2015 to 2016 States with good reporting had $80 \%$ to $<90 \%$ of drug overdose deaths mention of at least one specific drug in 2015 , with the change in the percentage of drug overdose deaths mentioning at least one specific drug differing by $<10$ percentage points from 2015 to 2016 . States included also were required to have stable rate estimates, based on $\geq 20$ deaths, in at least two drug categories (i.e., opioids, prescription opioids, synthetic opioids other than methadone, heroin, cocaine, and psychostimulants with abuse potential). South Dakota was the only state with good or excellent reporting in both years, but with an improvement $>10$ percentage points in drug specificity.

१ी Absolute rate change is the difference between 2015 and 2016 rates. Percent change is the absolute rate change divided by the 2015 rate, multiplied by 100. Nonoverlapping confidence intervals based on the gamma method were used if the number of deaths was $<100$ in 2015 or 2016 , and $z$-tests were used if the number of deaths was $\geq 100$ in both 2015 and 2016

*** Statistically significant at 0.05 level.

${ }^{+\dagger+}$ Cells with $\leq 9$ deaths are not reported. Rates based on $<20$ deaths are not considered reliable and not reported.

\section{Conflict of Interest}

No conflicts of interest were reported.

${ }^{1}$ Division of Unintentional Injury Prevention, National Center for Injury Prevention and Control, CDC; ${ }^{2}$ Epidemic Intelligence Service, CDC.

Corresponding authors: Puja Seth, pseth@cdc.gov, 404-639-6334; Lawrence Scholl, lzi8@cdc.gov, 404-498-1489.

\section{References}

1. Rudd RA, Seth P, David F, Scholl L. Increases in drug and opioidinvolved overdose deaths_-United States, 2010-2015. MMWR Morb Mortal Wkly Rep 2016;65:1445-52. https://doi.org/10.15585/mmwr. mm655051e1

2. Gladden RM, Martinez P, Seth P. Fentanyl law enforcement submissions and increases in synthetic opioid-involved overdose deaths - 27 states, 2013-2014. MMWR Morb Mortal Wkly Rep 2016;65:837-43. https:// doi.org/10.15585/mmwr.mm6533a2 


\section{Summary}

What is already known about this topic?

From 1999 to 2015, the drug overdose epidemic resulted in approximately 568,699 deaths. In 2015, 52,404 drug overdose deaths occurred; 63.1\% (33,091) involved an opioid. From 2014 to 2015 , the age-adjusted opioid-involved death rate increased by $15.6 \%$; the rapid increase in deaths was driven in large part by synthetic opioids other than methadone (e.g., fentanyl).

What is added by this report?

In 2016, there were 63,632 drug overdose deaths in the United States. Opioids accounted for $66.4 \%(42,249)$ of deaths, with increases across age groups, racial/ethnic groups, urbanization levels, and multiple states. Age-adjusted death rates for overdoses involving synthetic opioids other than methadone doubled from 2015 to 2016, and death rates from prescription opioids, heroin, cocaine, and psychostimulants also increased.

What are the implications for public health practice?

There is an urgent need to implement a multifaceted, collaborative public health and public safety approach. Building on existing resources, more rapidly available and comprehensive surveillance data are needed to track emerging drug threats to guide public action to prevent and respond to the epidemic through increased naloxone availability, harm reduction services, linkage into treatment (including medication-assisted treatment), safe prescribing practices, and supporting law enforcement strategies to reduce the illicit drug supply.
3. O’Donnell JK, Gladden RM, Seth P. Trends in deaths involving heroin and synthetic opioids excluding methadone, and law enforcement drug product reports, by census region-United States, 2006-2015. MMWR Morb Mortal Wkly Rep 2017;66:897-903. https://doi.org/10.15585/ mmwr.mm6634a2

4. Seth P, Rudd RA, Noonan RK, Haegerich TM. Quantifying the epidemic of prescription opioid overdose deaths. Am J Public Health 2018;108:500-2. https://doi.org/10.2105/AJPH.2017.304265

5. Rudd RA, Paulozzi LJ, Bauer MJ, et al. Increases in heroin overdose deaths_-28 states, 2010 to 2012. MMWR Morb Mortal Wkly Rep 2014;63:849-54.

6. O’Donnell JK, Halpin J, Mattson CL, Goldberger BA, Gladden RM. Deaths involving fentanyl, fentanyl analogs, and U-47700-10 states, July-December 2016. MMWR Morb Mortal Wkly Rep 2017;66:1197-202. https://doi.org/10.15585/mmwr.mm6643e1

7. McCall Jones C, Baldwin GT, Compton WM. Recent increases in cocaine-related overdose deaths and the role of opioids. Am J Public Health 2017;107:430-2. https://doi.org/10.2105/AJPH.2016.303627

8. Kandel DB, Hu MC, Griesler P, Wall M. Increases from 2002 to 2015 in prescription opioid overdose deaths in combination with other substances. Drug Alcohol Depend 2017;178:501-11. https://doi. org/10.1016/j.drugalcdep.2017.05.047

9. Davis GG; National Association of Medical Examiners and American College of Medical Toxicology Expert Panel on Evaluating and Reporting Opioid Deaths. Complete republication: National Association of Medical Examiners position paper: recommendations for the investigation, diagnosis, and certification of deaths related to opioid drugs. J Med Toxicol 2014;10:100-6. https://doi.org/10.1007/s13181-013-0323-x

10. Vivolo-Kantor AM, Seth P, Gladden RW, et al. Vital signs: trends in emergency department visits for suspected opioid overdoses-United States, July 2016-September 2017. MMWR Morb Mortal Wkly Rep 2018;67:279-85. https://doi.org/10.15585/mmwr.mm6709e1 